\title{
Finite-size scaling, intermittency and the QCD critical point
}

\author{
Nikolaos G. Antoniou \\ Faculty of Physics, University of Athens, GR-15784, Athens, Greece \\ E-mail: nantoniolphys.uoa.gr \\ Fotios K. Diakonos* \\ Faculty of Physics, University of Athens, GR-15784, Athens, Greece \\ E-mail: fdiakono@phys.uoa.gr
}

\begin{abstract}
Based on an effective action for the $3 \mathrm{~d}$ Ising critical point we construct a Ginzburg-Landau free energy for the proton density to describe the QCD thermodynamics in the critical region. We argue that the relation between finite-size scaling in configuration space and intermittency in momentum space is a valuable tool for the detection of the QCD critical point in ion collisions. We find that the critical region is very narrow along both chemical potential $(\mu)$ and temperature $(T)$ directions supporting that wide ranged beam energy scans with fixed size nuclei are unlikely to reach the critical region. Furthermore, we present a systematic procedure leading to the detection of the critical point through combined measurements of intermittency indices and freeze-out thermodynamic parameters $(\mu, T)$ for protons. Exploiting previous NA49 measurement of the intermittency index $\phi_{2}$ and the freeze-out parameters $(\mu, T)$ for protons produced in central $\mathrm{Si}+\mathrm{Si}$ " collision at $158 \mathrm{~A} \mathrm{GeV}$ we predict the approach to the critical point in peripheral $\mathrm{Ar}+\mathrm{Sc}$ collisions of the NA61/SHINE experiment at maximum SPS (CERN) energy.
\end{abstract}

Corfu Summer Institute 2018 "School and Workshops on Elementary Particle Physics and Gravity" (CORFU2018)

31 August - 28 September, 2018

Corfu, Greece

${ }^{*}$ Speaker. 


\section{Introduction}

The detection of the QCD critical point, remnant of the chiral transition at high temperatures and finite chemical potential, is the highest priority goal of recent, current and forthcoming ultra-relativistic ion collision experiments. So far, due to the well known sign problem, Lattice QCD calculations do not provide an unambiguous result for its existence and location. Thus, phenomenological procedures which could guide the systematic approach to the critical point, exploiting experimental data, are very welcome. Such a strategy is developed in this work. It is based on theoretical arguments, suggesting that the QCD critical point appears as the endpoint (at low baryochemical potential) of a continuous line of first order transitions, belonging to the $3 \mathrm{~d}$-Ising universality class [1]. Having fixed the universality class the next important step is the determination of the associated order parameter. The chiral condensate or sigma field $\sigma=\langle\bar{q} q\rangle$ plays this role, however, it is not directly observable in ion collision experiments. Since the strong interaction mixes $\sigma$ with protons, the critical fluctuations of the former are transferred to the proton density [2] as well. Thus, proton density fluctuations contain a critical sector and due to net baryon conservation they form the slow component in the dynamics of the QCD order parameter [3]. Combining the knowledge of the universality class (3d-Ising) and the suitable order parameter (proton density) with the fact that the effective action describing the critical thermodynamics of the 3d-Ising magnet is accurately calculated [4], one can obtain a free-energy for the proton density close to the QCD critical point [5]. Furthermore, employing this free energy and the associated, Ising model based, QCD partition function $\mathscr{Z}_{I Q C D}$, one can calculate a set of quantities with a characteristic behaviour when approaching the critical point. From experimental point of view a very interesting and easily accessible quantity is the second factorial moment of protons $F_{2}(M)$ calculated in $M^{2}$ small cells partitioning the associated transverse momentum space. When the protons are produced from a fireball freezing out close enough to the critical point, so that finite-size scaling (FSS) applies, then the corresponding $F_{2}(M)$ attains a power-law form $F_{2}(M) \sim M^{2 \phi_{2}}$ with $\phi_{2}$ determined by the isothermal critical exponent $\delta$ of the 3d-Ising ferromagnetic transition [6]. This effect, called intermittency, is the analogue of critical opalescence in microscopic physics involving particle production. The gradual destruction of this power-law, as the produced fireball freeze-out state departs from the immediate neighbourhood of the critical point, can be used as a tool to detect the proximity to the QCD critical point. In the next sections we will describe how this can be realized in practice. Our analysis shows that the critical region is very narrow along the baryochemical potential $(\mu)$ and temperature $(T)$ directions which disfavours its search using wide ranged beam energy scans with fixed size colliding nuclei. Furthermore, we will exploit existing measurements of $F_{2}(M)$ for protons produced in central $\mathrm{Si}+" \mathrm{Si}$ " collisions at 158A GeV (NA49 experiment, SPS, CERN) to estimate the critical point location and predict the existence of critical fluctuations in peripheral $\mathrm{Ar}+\mathrm{Sc}$ collisions of the NA61/SHINE experiment (SPS, CERN) at highest colliding energy (150A $\mathrm{GeV})$.

\section{Ising-QCD thermodynamics in the critical region}

In this section our goal is to construct first a free-energy for the description of the proton fluid thermodynamics close to the QCD critical point. Starting point is the 3d-Ising effective action $S_{\text {eff }}$ 
for the order parameter $\phi$ in the critical region [4]. The field $\phi$ is given as $\phi=\beta_{c}^{3} \lim _{\delta V \rightarrow 0} \frac{n_{\uparrow}-n_{\downarrow}}{\delta V}$ where $n_{\uparrow}\left(n_{\downarrow}\right)$ is the number of spins in the up (down) direction within a space cell of volume $\delta V$, while $\beta_{c}$ is the inverse temperature, used to make $\phi$ dimensionless. Thus, in dimensionless form $S_{\text {eff }}$ is written as:

$$
\begin{aligned}
S_{e f f} & =\int_{V} d^{3} \hat{\mathbf{x}}\left[\frac{1}{2}|\hat{\nabla} \phi|^{2}+U(\phi)-\hat{h} \phi\right] \\
U(\phi) & =\frac{1}{2} \hat{m}^{2} \phi^{2}+\hat{m} g_{4} \phi^{4}+g_{6} \phi^{6}
\end{aligned}
$$

with $\hat{x}=x \beta_{c}^{-1}$ the spatial coordinate, $\hat{m}=\beta_{c} m=\beta_{c} \xi^{-1}$ the inverse correlation length $(\xi)$ and $\hat{h}=h \beta_{c}^{-1}$ the ordering field, made dimensionless through the length scale $\beta_{c}$. The dimensionless constants $g_{4} \approx 0.97, g_{6} \approx 2.1$ are universal in the $3 \mathrm{~d}$-Ising class. The temperature $T$ enters in eq. (2.1) through the correlation length $\xi$ given as $\xi=\xi_{0, \pm}\left|1-\frac{T}{T_{c}}\right|^{-v}$ with $\xi_{0, \pm}$ non-universal but with a universal ratio $\frac{\xi_{0,+}}{\xi_{0,-}}=2$. Notice that for the $3 \mathrm{~d}$-Ising $v=\frac{2}{3}$. The corresponding partition function $\mathscr{Z}$ is obtained summing over small wavelength configurations $\{\phi\}$ as $\mathscr{Z}=\sum_{\{\phi\}} \exp \left(-S_{\text {eff }}[\phi]\right)$.

To construct the Ising-QCD partition function in the critical region we have to map the 3d-Ising variables to the corresponding variables of QCD. This is straightforward for the order parameter using the map: $\left(n_{\uparrow}, n_{\downarrow}\right) \Longrightarrow\left(n_{B}, n_{\bar{B}}\right)$ with $n_{B}\left(n_{\bar{B}}\right)$ the number of baryons (antibaryons) with the cell $\delta V$. To complete the mapping, one has to determine the relation between the $3 \mathrm{~d}$-Ising variables $(h, T)$ and the QCD thermodynamic variables $(\mu, T)$ (where $\mu$ is the baryochemical potential). In general this mapping is not uniquely determined [7]. In fact one can write:

$$
\begin{aligned}
& h \rightarrow\left(\mu-\mu_{c}\right)-\tan \alpha\left(T-T_{c}\right) \\
& \xi \rightarrow \xi_{0, \pm}\left|\frac{T}{T_{c}}-1+\tan \alpha \frac{\left(\mu-\mu_{c}\right)}{T_{c}}\right|^{-v}
\end{aligned}
$$

with $\alpha$ a free parameter. Here we will focus on the case $\alpha=0$. However, the obtained results are robust and remain practically unchanged up to $\alpha \approx 60^{\circ}$. Only for $\alpha \approx 90^{\circ}$ occur some peculiarities which will be discussed later on.

Following [2] we adopt the scenario that proton density fluctuations have a similar description as in eq. (2.1). Then, using constant configurations for the field $\phi=\frac{N_{p}}{V}\left(N_{p}\right.$ is the number of protons) we can write the Ising-QCD partition function for the protons, within the critical region, as:

$$
\mathscr{Z}_{I Q C D}=\sum_{N=0}^{\Lambda} \zeta^{N} \exp \left[-\frac{1}{2} \hat{m}^{2} \frac{N^{2}}{\Lambda}-g_{4} \hat{m} \frac{N^{4}}{\Lambda^{3}}-g_{6} \frac{N^{6}}{\Lambda^{5}}\right]
$$

We use the notation $\zeta=\exp \left[\left(\mu-\mu_{c}\right) \beta_{c}\right]$ and $\Lambda=\frac{V}{V_{0}}$, where $V_{0}$ is the volume of a single proton.

Having determined $\mathscr{Z}_{I Q C D}$ we can use it to:

- Calculate proton multiplicity moments $\left\langle N^{k}\right\rangle(k=1,2, .$.$) and check if they follow a power-$ law, as a function of the system's size, with critical exponents compatible with the 3-d Ising universality class. In particular, we can also consider the variation of the dependence of the multiplicity moments on system's size, as the distances form the critical point $m u_{B}-\mu_{c}$ and 
$T-T_{c}$ increase. This could provide us with information related to the size of the critical region.

- Calculate thermodynamic response functions like specific heat, susceptibility, etc. And, finally,

- determine the equation of state, calculating the pressure, in the neighbourhood of the critical point.

In the next section we will focus on the first of these tasks.

\section{Finite-size scaling, proton multiplicity moments and intermittency}

We first calculate the proton multiplicity moments $\left\langle N_{p}^{k}\right\rangle$ using the partition function (2.3). To simplify the notation we use $N$ instead of $N_{p}$ in the following. Exactly at the critical point $\left(\mu=\mu_{c}\right.$, $T=T_{c}$ ) we find:

$$
\left\langle N^{k}\right\rangle \sim \Lambda^{k q}, \quad q=d_{F} / d, \quad k=1,2, . .
$$

This result can be understood in terms of finite size scaling (FSS). The finite critical system is characterized by proton density fluctuations forming a fractal set with dimension $d_{F}=\frac{5}{2}$ embedded in the three dimensional space. the FSS exponent $q$ is related to the isothermal critical exponent $\delta$ [5]:

$$
q=\frac{d_{F}}{d}=\frac{\delta}{\delta+1} \quad ; \quad \delta=5(3 \mathrm{~d}-\text { Ising })
$$

Thus, a measurement of $q$ corresponds to a measurement of $\delta$. However, it is unrealistic task to perform this measurement guided by equation (3.1) since in this case one needs systems of different sizes freezing out at the critical point.

Nevertheless, since in the FSS regime the local scaling:

$$
\left\langle n(\mathbf{x}) n\left(\mathbf{x}^{\prime}\right)\right\rangle \sim\left|\mathbf{x}-\mathbf{x}^{\prime}\right|^{-\left(3-d_{F}\right)}
$$

is valid also globally $\left(\left|\mathbf{x}-\mathbf{x}^{\prime}\right|=O\left(V^{1 / 3}\right)\right)$, a large distance singular behaviour of the density-density correlator $\langle N\rangle \propto \Lambda^{\frac{d_{F}}{3}}$ is established. This singular behaviour is transferred to the density-density correlation in proton transverse momentum space, for small momentum differences [8]:

$$
\lim _{\mathbf{k} \rightarrow \mathbf{k}^{\prime}}\left\langle n(\mathbf{k}) n\left(\mathbf{k}^{\prime}\right)\right\rangle \sim\left|\mathbf{k}-\mathbf{k}^{\prime}\right|^{-2 q} \quad ; \quad q=\frac{d_{F}}{3}=\frac{5}{6}
$$

Such a singularity is detectable through intermittency (critical opalescence) in proton transverse momentum space [6] allowing the measurement of $q$ and therefore also the measurement of the isothermal critical exponent $\delta[8]$ !

Another quantity which is related to proton multiplicity moments and it is widely used in the search of the critical point is the non-Gaussian kurtosis, defined as:

$$
\kappa_{n G}=\frac{C_{4}-3 C_{2}^{2}}{C_{2}^{2}} ; \quad C_{k}=\left\langle(N-\langle N\rangle)^{k}\right\rangle, \quad k=2,3, . .
$$



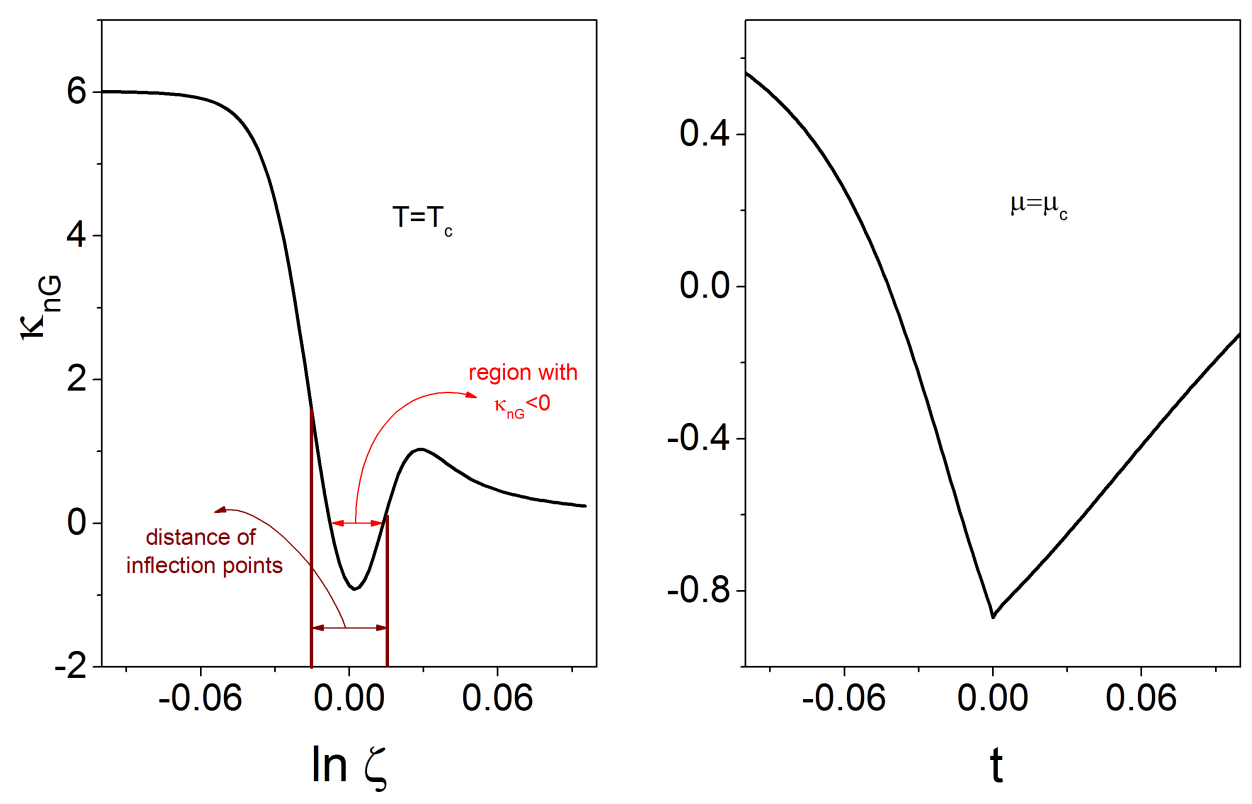

Figure 1: The left plot shows $\kappa_{n G}(\ln \zeta)$ for $t=0$. The right plot shows $\kappa_{n G}(t)$ for $\ln \zeta=0$.

It is argued that $\kappa_{n G}$ possesses a non-monotonic behaviour attaining a negative minimum when crossing the critical point $[9,11]$. One can use $\mathscr{Z}_{I Q C D}$ to calculate the proton multiplicity cumulants $C_{k}$ and $\kappa_{n G}$ through the relations:

$$
\frac{\partial^{2}}{\partial(\ln \zeta)^{2}} \ln \mathscr{Z}_{I Q C D}=C_{2} \quad ; \quad \frac{\partial^{4}}{\partial(\ln \zeta)^{4}} \ln \mathscr{Z}_{I Q C D}=C_{4}-3 C_{2}^{2}
$$

and explore their behaviour close to the critical point. The result of this calculation is illustrated in Fig. 1.

In the left plot we show $\kappa_{n G}$ versus $\ln \zeta$ around the critical point $(\ln \zeta=0)$ while in the right plot we show the function $\kappa_{n G}(t)$ around $t=0$, with $t$ the reduced temperature $t=\frac{T-T_{c}}{T_{c}}$. We clearly observe the emergence of a sharp, negative minimum close to the critical point. In the next section we will use this behaviour to determine the size of the critical region.

\section{Size of the critical region}

In this section we estimate the size of the critical region using two different approaches: (i) exploring the gradual destruction of the FSS law $\langle N\rangle \sim \Lambda^{\frac{5}{6}}$ (see eq. (3.1) when departing from the critical point $(\zeta=1, t=0)$ and (ii) estimating the region in the $(\ln \zeta, t)$-plane, for which $\kappa_{n G}$ becomes negative.

We present first a plot (see Fig. 2) demonstrating the destruction of the FSS law which occurs when $(\zeta, t)$ depart from the critical values $(1,0)$. 

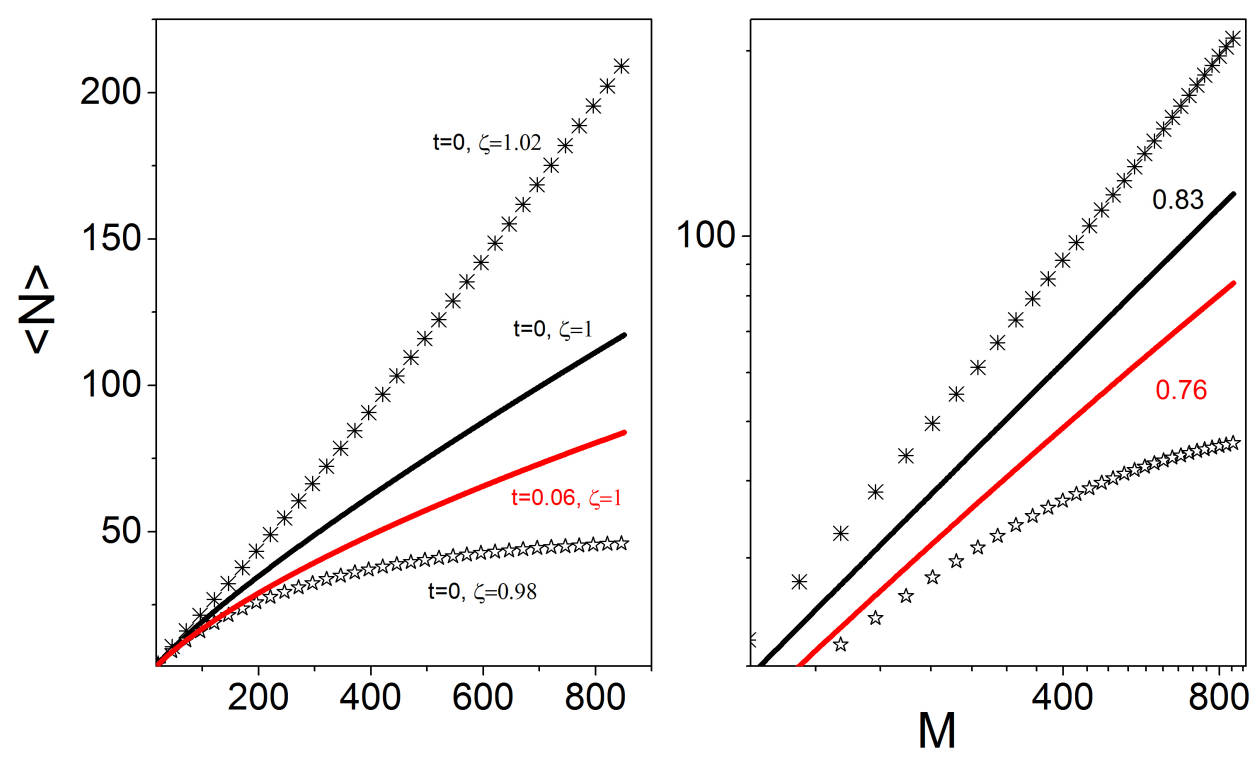

Figure 2: The left plot shows $\langle N\rangle$ for different values of $\ln \zeta$ and $t$ close to the critical point $(0,0)$. The right plot is the same as the left plot in double logarithmic scale.

The right plot is the same as the left plot in double logarithmic scale. It clearly demonstrates that in a region close to $(1,0)$ a modified power-law:

$$
\langle N\rangle \sim \Lambda^{\tilde{q}}
$$

is holding. When $\tilde{q}$ becomes 1 , the scaling valid for conventional systems is established, while when $\tilde{q}=\frac{3}{4}$, the scaling behaviour is that of the mean field theory $(\delta=3)$. Thus, a reasonable choice for the definition of the critical region is the subspace of the $(\ln \zeta, t)$-plane for which $\frac{3}{4}<\tilde{q}<1$ is valid. This condition restricts significantly the size of the critical region along the baryochemical direction leading to $\Delta \mu \approx 5 \mathrm{MeV}$ [5] assuming a critical temperature $T_{c} \approx 160 \mathrm{MeV}$. The later is compatible with recent Lattice QCD results [12]. In Fig. 3 we show the critical (red shaded) region in the $(\ln \zeta, t)$ plane, estimated with the condition $\frac{3}{4}<\tilde{q}<1$. In the plot the grey point is the critical one with coordinates $(0,0)$. We add in the plot the blue line consisting of $(\ln \zeta, t)$-pairs which lead to a scaling compatible with eq. (4.1) for $\tilde{q}=0.96$. The reasons for adding this line will be clarified later in this work.

A strong restriction along the $t$-direction occurs when we require additionally the validity of FSS: $\xi_{\infty}>V^{1 / 3}$ with $\xi_{\infty}=\xi_{0, \pm}\left|\frac{T-T_{c}}{T_{c}}\right|$ the infinite system correlation length and $V$ the volume of the finite system. For medium size nuclei $(20<A<50)$ we find $3 \mathrm{MeV}<\Delta T<5 \mathrm{MeV}$ for the extension of the FSS region along the temperature axis [10].

We consider now an alternative way to define the size of the critical region, determining the region of the $(\ln \zeta, t)$ plane which leads to negative values for $\kappa_{n G}$. We use the opportunity to check 


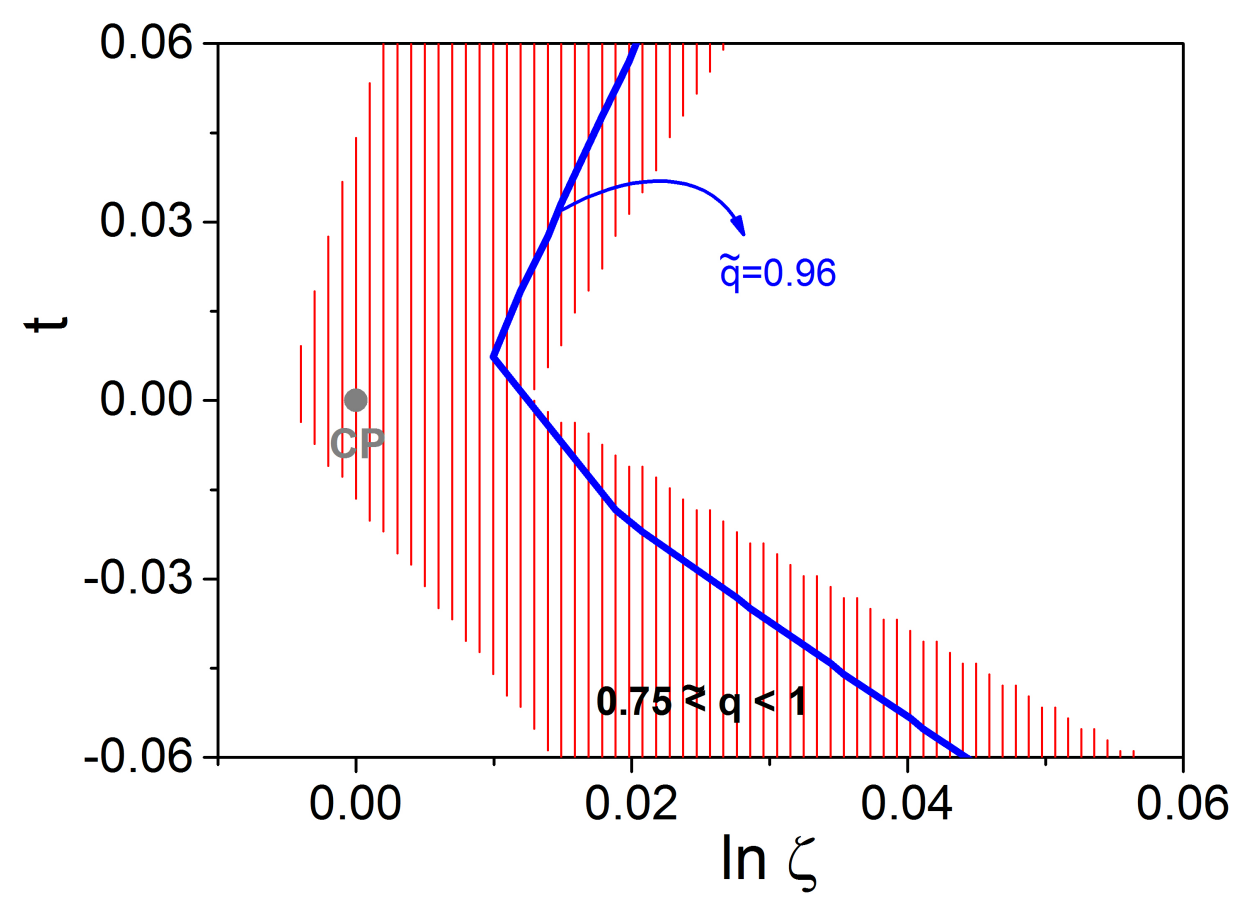

Figure 3: The critical region (red shaded area) in the $(\ln \zeta, t)$ plane determined through the condition $\frac{3}{4}<$ $\tilde{q}<1$.

also the impact of $\alpha$ in the map (2.2). The results of these calculations are given in Fig. 4 where the red shaded area displays the critical region estimated through the negativity of $\kappa_{n} G$ while the blue shaded region shows the corresponding result using the condition $\frac{3}{4}<\tilde{q}<1$. For $\alpha=0$ (left upper plot) the two methods lead to very similar results. A slight difference is observed for $\alpha=45^{\circ}$ (right upper plot) which is significantly increased for $\alpha=93^{\circ}$ (lower plot).

We define as critical region the cut between the two sets. According to this definition the critical-region size along $\mu$ is $3 \mathrm{MeV} \leq \Delta \mu \leq 11 \mathrm{MeV}$ for all values of $\alpha$ [10]. This results disfavours the search for the critical point using ion collisions with fixed size nuclei colliding with energy varying in large steps [11].

\section{Locating the CEP}

In this section we show how the theoretical framework described previously, combined with the intermittency analysis results and knowledge of $(\mu, T)$ for the fireball's freeze-out state, enables to locate the critical point in the $(\mu, T)$-plane. The key observation in this approach is that the final state created in $\mathrm{Si}+\mathrm{Si}$ " central collisions at $\sqrt{s}=17.2 \mathrm{GeV}$ lies within the critical region since the associated intermittency analysis gives $\tilde{q} \approx 0.96$ [13]. Thus, the freeze-out parameters $\left(\mu_{S i}, T_{S i}\right)$ for $\mathrm{Si}+" \mathrm{Si}$ " should lead to $(\ln \zeta, t)$ values lying on the blue line in Fig. 3 . In fact both, the $\tilde{q}$ measurement as well as the estimation of $\left(\mu_{S i}, T_{S i}\right)$ [14], contain large errors giving to the subsequent treatment mainly indicative character. To proceed, we are going to neglect all these 

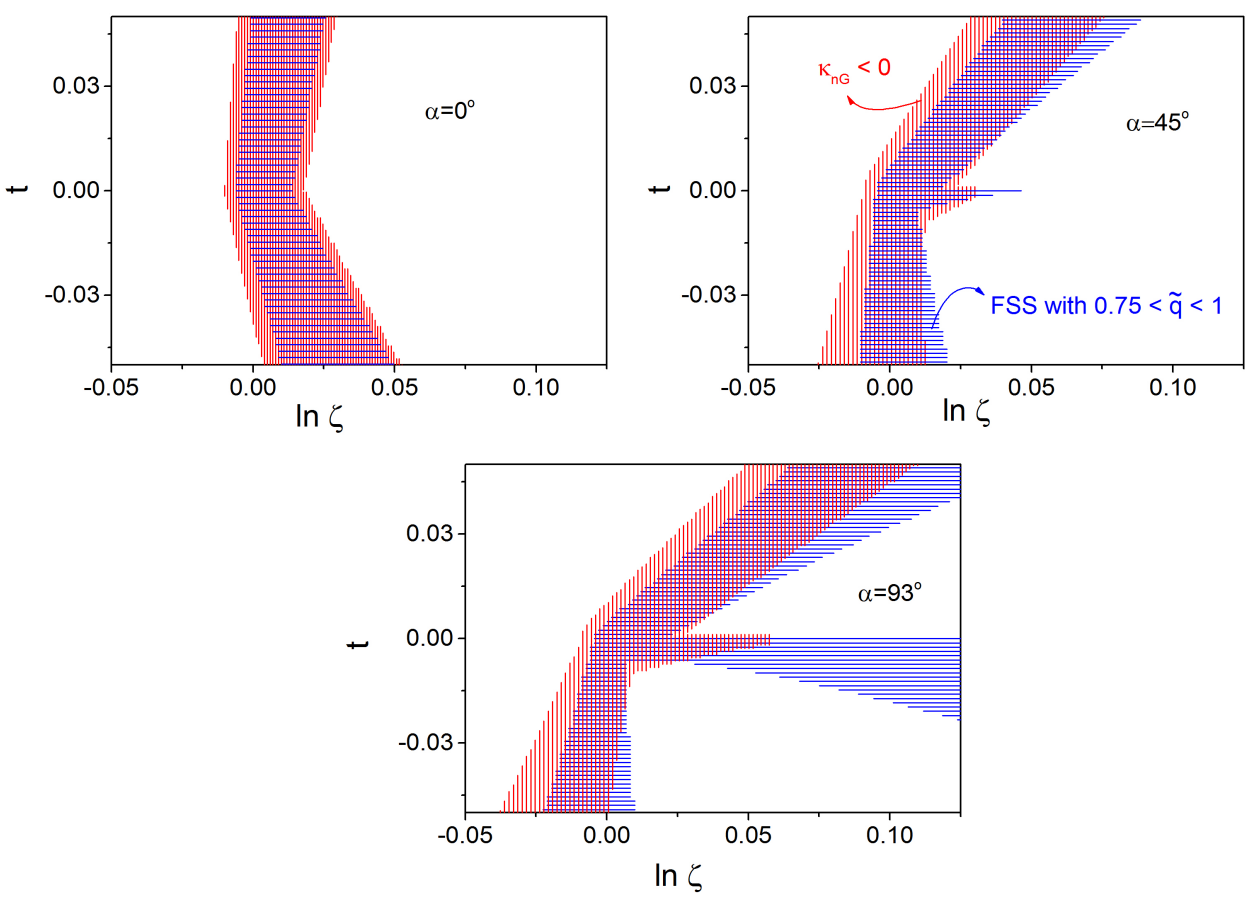

Figure 4: The critical region for various values of $\alpha$ calculated with two methods: (i) as the $(\ln \zeta, t)$ subspace for which $\langle N\rangle \sim \Lambda^{\tilde{q}}$ with $\frac{3}{4}<\tilde{q}<1$ (blue shaded region) and (ii) the $(\ln \zeta, t)$ domain for which $\kappa_{n G}<0$ (red shaded region). In the upper left plot $\alpha=0^{\circ}$, in the upper right plot $\alpha=45^{\circ}$ and in the lower plot $\alpha=93^{\circ}$.

errors and therefore the obtained qualitative results should be considered with caution. Given $T_{c}$, the line $\tilde{q}=0.96$ determines $\mu_{c}$, provided that the values $\left(\mu_{S i}, T_{S i}\right)$ are known. Here we use $T_{c}=163$ $\mathrm{MeV}$ which is compatible with the Lattice QCD estimation in [12] and $\left(\mu_{S i}, T_{S i}\right)=(260,162.2)$ $\mathrm{MeV}$, being the central values obtained in [14], which lead to $\ln \zeta_{S i}=0.0143$ for locating the $\mathrm{Si}+" \mathrm{Si}$ " freeze-out state on the blue line of Fig. 3. This in turn leads to $\mu_{c}=257.7 \mathrm{MeV}$.

Using for the critical point location the values $\left(\mu_{c}, T_{c}\right)=(257.7,163) \mathrm{MeV}$ it is possible to make some predictions concerning the intermittency results ( $\phi_{2}$ or $\tilde{q}$ measurements) expected for $\mathrm{Ar}+\mathrm{Sc}$ system in NA61/SHINE experiment. To achieve this we need the corresponding freeze-out parameters $\left(\mu_{A r S c}, T_{A r S c}\right)$. They can be estimated with the help of the NA49 freeze-out parameters for the $\mathrm{C}+\mathrm{C}, \mathrm{Si}+\mathrm{Si}$ " and $\mathrm{Pb}+\mathrm{Pb}$ systems (central collisions at $\sqrt{s}=17.2 \mathrm{GeV}$ ) given in [14], employing a suitable parametrization for the functions $T(A)$ and $\mu(A)$ with $A$ the mass number of the colliding nuclei. In Fig. 5 we show the results for such a procedure, described in detail in [10].

For central collisions at $\sqrt{s}=17.2 \mathrm{GeV}$, we find $\left(\mu_{A r S c}, T_{A r S c}\right)=(258,160.9) \mathrm{MeV}$ and $\left(\mu_{X e L a}, T_{X e L a}\right)=(251,158.2) \mathrm{MeV}$. One could now try to place these freeze-out states on the critical region plot of Fig. 3. Due to the narrowness of the critical region only the Ar+Sc system fits in this plot. The enriched diagram of the critical region is shown in Fig. 6. We observe that the fireball formed in central Ar+Sc collisions lies close but outside of the corresponding FSS region within a distance of $\Delta T \approx 1 \mathrm{MeV}$ along the temperature axis. This opens up the perspective that the freeze-out states of peripheral Ar+Sc enter into the FSS region. 

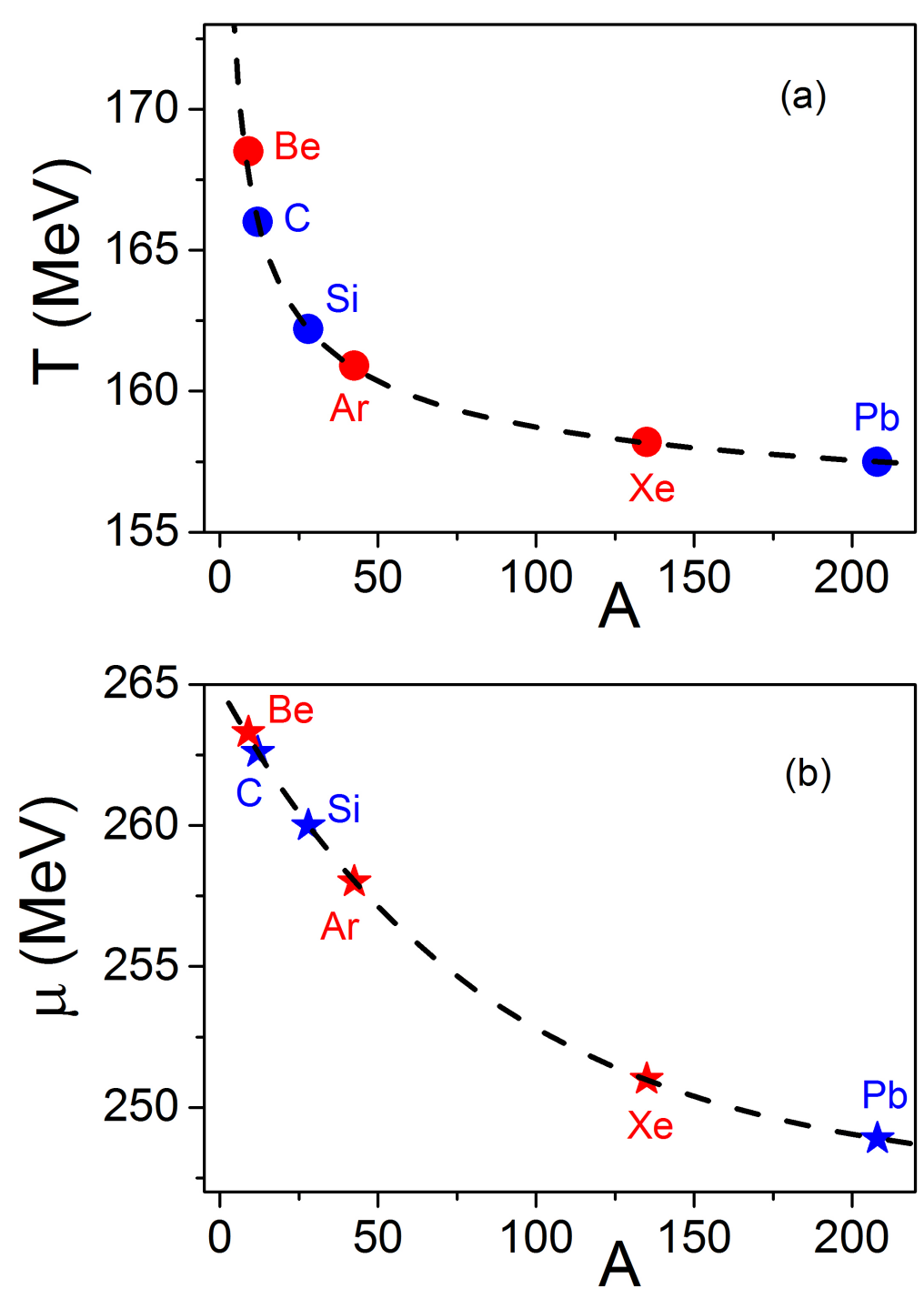

Figure 5: The functions $T(A)$ and $\mu(A)$ for central $\mathrm{A}+\mathrm{A}$ collisions at $\sqrt{s}=17.2 \mathrm{GeV}$ colliding energy. The values for the NA49 systems $(\mathrm{C}, \mathrm{Si}, \mathrm{Pb})$ are obtained from [14] while those of the NA61/SHINE systems $(\mathrm{Be}, \mathrm{Ar}, \mathrm{Xe})$ are obtained by a suitable interpolation.

\section{Conclusions}

Summarizing our results, we have found that:

- Critical (FSS) region is very narrow $(5-10 \mathrm{MeV})$ along the $\mu$ and the $T$ axis. This makes very unlike the approach to the critical region with beam energy scan involving fixed size nuclei and large energy steps. 


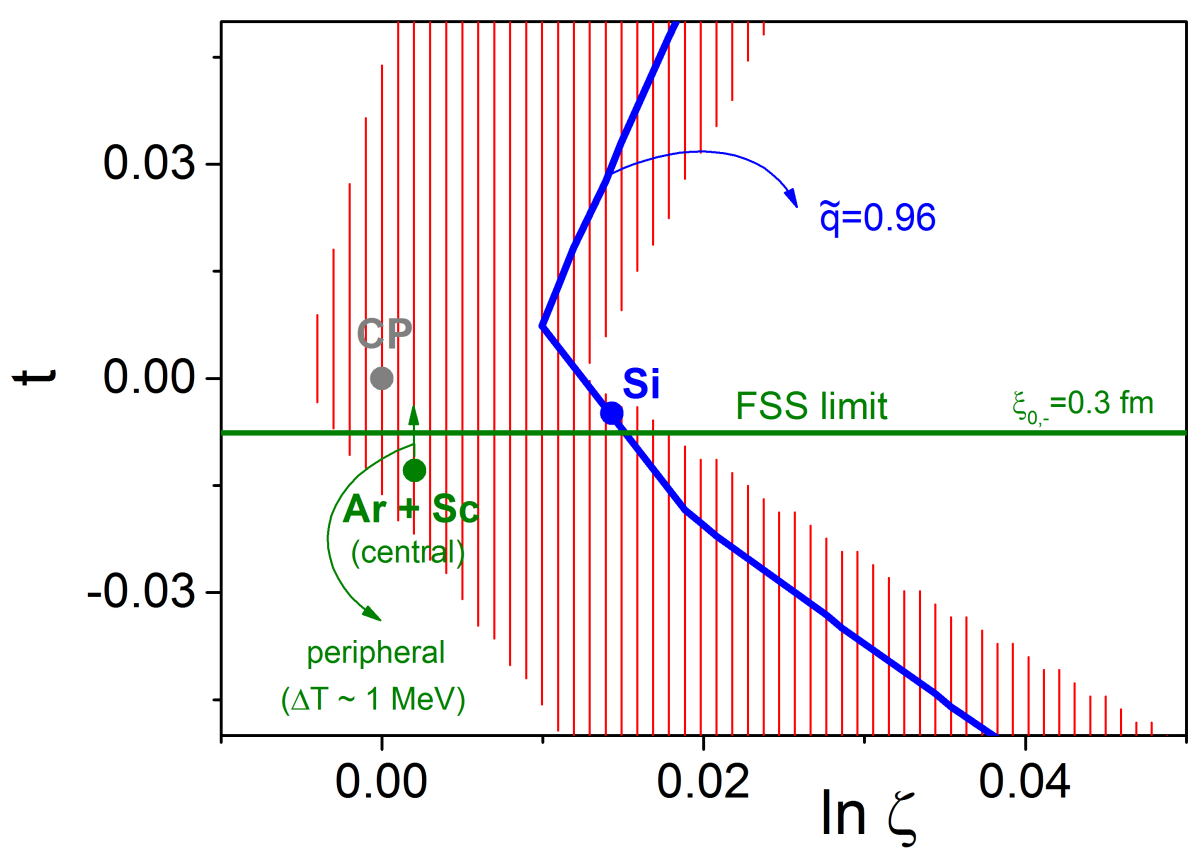

Figure 6: A sketch of the critical region (red shaded area) in the $(\ln \zeta, t)$ plane as in Fig. 3, enriched with the freeze-out states of $\mathrm{Si}+\mathrm{Si}$ " (NA49 experiment) and Ar+Sc (NA61/SHINE experiment) produced through central collisions at $\sqrt{s}=17 \mathrm{GeV}$.

- Ignoring errors the freeze-out state of central $\mathrm{Si}+\mathrm{Si}$ collisions at $\sqrt{s}=17.2 \mathrm{GeV}$ lies within the critical (FSS) region. This is an important result of NA49 experiment (SPS, CERN) which can be used as a guide for detecting the QCD critical point.

- A promising strategy for the detection of the critical point is to combine accurate measurements of FSS exponent $\tilde{q}$ (intermittency analysis) and corresponding freeze-out parameters $(\mu, T)$ in central $\mathrm{A}+\mathrm{A}$ collisions with $25<\mathrm{A}<50$ and in peripheral collisions with $32<\mathrm{A}<50$.

- The collision energy $\sqrt{s} \approx 17 \mathrm{GeV}$ seems to be the appropriate for approaching $\mu_{c}$. Peripheral collisions can be used for fine changes in $T$ allowing the entrance into the FSS region.

As a consequence we predict strong intermittency effect in peripheral $\mathrm{Ar}+\mathrm{Sc}$ collisions at $\sqrt{s} \approx 17 \mathrm{GeV}$ (NA61/SHINE experiment) [10].

\section{References}

[1] S. Gavin, A. Gocksch and R. D. Pisarski, Phys. Rev. D 49, 3079 (1994); M. Stephanov, K. Rajagopal and E. Shuryak, Phys. Rev. Lett. 81, 4816 (1998); M. A. Halasz, A. D. Jackson, R. E. Shrock, M. A. Stephanov and J. J. M. Verbaarschot, Phys. Rev. D 58, 096007 (1998); J. Berges and K. Rajagopal, Nucl. Phys. B 538, 215 (1999). 
[2] Y. Hatta and M. A. Stephanov, Phys. Rev. Lett. 91, 102003 (2003).

[3] Y. Minami, Phys. Rev. D 83, 094019 (2011).

[4] M. M. Tsypin, Phys. Rev. Lett. 73, 2015 (1994).

[5] N. G. Antoniou, F. K. Diakonos, X. N. Maintas and C. E. Tsagkarakis, Phys. Rev. D 97, 034015 (2018).

[6] N. G. Antoniou, F. K. Diakonos, A. S. Kapoyannis and K. S. Kousouris, Phys. Rev. Lett. 97, 032002 (2006).

[7] J. J. Rehr and N. D. Mermin, Phys. Rev. A 8, 472 (1973).

[8] N. G. Antoniou, N. Davis, F. K. Diakonos, Phys. Rev. C 93, 014908 (2016).

[9] M. A. Stephanov, Phys. Rev. Lett. 102, 032301 (2009); M. A. Stephanov, Phys. Rev. Lett. 107, 052301 (2011).

[10] N. G. Antoniou and F. K. Diakonos, J. of Phys. G 46, 035101 (2019).

[11] N. G. Antoniou, F. K. Diakonos, N. Kalntis and A. Kanargias, Nucl. Phys. A 986, 167 (2019).

[12] S. Datta et al, Phys. Rev. D 95, 054512 (2017).

[13] T. Anticic et al., Eur. Phys. J. C 75, 587 (2015).

[14] F. Becattini, J. Manninen and M. Gazdzicki, Phys. Rev. C 73, 044905 (2006). 